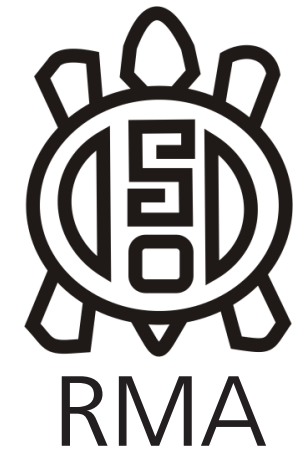

Antropología Social

\title{
Por una Sociología de carne y sangre
}

\author{
For a Sociology of Flesh and Blood
}

El presente texto se publicó en simultáneo en alemán (Berliner Debatte Initial and Sub/Urban), danés (Grunde), noruego (Sosiologisk Tidsskrift), finés (Sosiologia), turco (Cogito), inglés (Qualitative Sociology), francés (Terrains et travaux), Rumano (Compaso), Coreano (Sahoi gwahak yeongu) e Italiano. Esta es la primera versión en español.

Loïc Wacquant

Departamento de Sociología, University of California, Berkeley. Email: loic@berkeley.edu

Traducido por Diego Roldán (CONICET) y Paul Hathazy (CIECS CONICET/UNC)

\begin{abstract}
Resumen
Este artículo elabora la ontología social y la metodología de la sociología carnal como un modo distintivo de indagación social, evitando la postura espectadora para captar la acción-en-proceso, a raíz de los debates desencadenados por mi estudio basado en el aprendizaje del boxeo como un arte corporal plebeyo. Primero critico las nociones de agente (dualista), estructura (externalista) y conocimiento (mentalista) que prevalecen en las ciencias sociales contemporáneas y esbozo una concepción alternativa del animal social, no solo como portadora de símbolos, sino como una criatura de carne y sangre, sensible, sufirente, hábil, sedimentada y situada criatura de carne y hueso. Destaco la primacía de los conocimientos prácticos incorporados que surgen y se enredan continuamente en redes de acción y considero qué modos de investigación son adecuados para desplegar y extraer esta concepción encarnada del agente. Sostengo que la etnografía performativa, la marca del trabajo de campo inmersivo basado en "realizar el fenómeno", es un camino fructífero hacia la captura de los esquemas (habitus) cognitivos, conativos y catécticos que generan las prácticas y subyacen en el cosmos que se está investigando. Pero se necesita talento social y persistencia para obtener los beneficios de la "participación observante" y lograr la competencia social (a diferencia de la saturación empírica). Para concluir, vuelvo al diálogo de Bourdieu con Pascal para considerar la dificultad y la urgencia especiales de capturar el "espíritu de agudeza" que anima dicha competencia pero desaparece de los relatos sociológicas normales.
\end{abstract}

Palabras clave: Acción; Estructura; Conocimiento; Cuerpo; Encarnación; Habitus; Ontología social; Participación observante; Etnografía enactiva; Bourdieu; Pascal.

\begin{abstract}
This article elaborates the social ontology and methodology of carnal sociology as a distinctive mode of social inquiry eschewing the spectatorial posture to grasp action-in-the-making, in the wake of debates triggered by my apprenticeship-based study of boxing as a plebeian bodily craft. First I critique the notions of (dualist) agent, (externalist) structure, and (mentalist) knowledge prevalent in the contemporary social sciences and sketch an alternative conception of the social animal, not just as wielder of symbols, but as sensate, suffering, skilled, sedimented, and situated creature of flesh and blood. I spotlight the primacy of embodied practical knowledge arising out of and continuously enmeshed in webs of action and consider what modes of inquiry are suited to deploying and mining this incarnate conception of the agent. I argue that enactive ethnography, the brand of immersive fieldwork based on "performing the phenomenon," is a fruitful path toward capturing the cognitive, conative, and cathectic schemata (habitus) that generate the practices and underlie the cosmos under investigation. But it takes social spunk and persistence to reap the rewards of "observant participation" and achieve social competency (as distinct from empirical saturation). In closing, I return to Bourdieu's dialogue with Pascal to consider the special difficulty and urgency of capturing the "spirit of acuteness" that animates such competency but vanishes from normal sociological accounts. [As published in Loïc Wacquant Qual. Sociol. (2015) 38:1-11]
\end{abstract}

Keywords: Action; Structure; Knowledge; Body; Incarnation; Habitus; Social Ontology; Observant participation; Enactive ethnography; Bourdieu; Pascal.

Recibido 20-02-2018. Recibido con correcciones 26-02-2019. Aceptado 24-03-2019 
Este artículo es una versión ampliada de un texto escrito originalmente en respuesta a una serie de preguntas que me efectuó Frank Adloff (sociólogo en la FriedrichAlexander-Universität en Núremberg y en el Instituto Universitario Europeo de Florencia) y algunos de sus colegas para un volumen colectivo que explora la relación entre "corporización y explicación" en el análisis social (Adloff et al. 2014). Estas consultas son utilizadas como un trampolín para aclarar cuestiones planteadas en una serie de debates acerca de la teoría bourdesiana de la acción disposicional, con la que me he involucrado durante la última década, en varias conferencias, así como en publicaciones, tras mi investigación carnal sobre el boxeo como arte corporal plebeyo (ver, en particular, el número especial de Qualitative Sociology sobre Body and Soul, summer 2005, Auyero (2005), y los simposios sobre "Habitus in Body \& Soul", en Theory and Psicology, December 2009, Henderikus (2009) y "Hominies in Extremis", Body \& Society, spring 2014, así como Wacquant 2009)

Primero, critico las nociones de agente (dualista), estructura (externa lista) y conocimiento (idealista) predominantes en las ciencias sociales contemporáneas y bosquejo una concepción alternativa del animal social. Propongo caracterizar a este último no sólo como un creador neo-kantinao y portador de símbolos -en la línea de Ernst Cassirer, George Herbert Mead y John Searle (2009, IX), para quienes los humanos son "conscientes, racionales, performadores de actos de habla (speech-act performing), poseedores de libre albedrío, seres sociales y políticos"- sino también como una criatura corporal sensible, sufrida, hábil, sedimentada y situada. Destaco la primacía del conocimiento práctico incorporado que surge de y se enreda continuamente en las redes de acción, sobre las cuales se injerta el dominio discursivo. Luego considero qué modos de investigación están en condiciones de desarrollar y extraer esta concepción encarnada del animal humano. Argumento que la etnografía enactiva, el tipo de trabajo de campo de inmersión, basado en "performar el fenómeno", es un camino fructífero para revelar los esquemas cognoscitivos, conativos y catécticos (es decir, el habitus) que generan las prácticas y subyacen al cosmos bajo investigación. Pero se necesita talento social y persistencia para excavar en una posición adecuada de "participación con observación" y cosechar sus recompensas. Especificar la ontología social y la metodología de la sociología carnal me conduce a retomar el diálogo de Bourdieu con Pascal para considerar la dificultad específica y la urgencia de capturar el "espíritu vital" que anima la competencia social, pero que es borrado en los relatos sociales y científicos normales.

¿Qué tan importante es para usted enfocarse en las dimensiones implícitas (conocimiento tácito, saber hacer, sentido práctico, etc.) de la interacción social?

Es crucial si queremos superar tres errores perennes que obstaculizan las ciencias sociales y nos impiden desarrollar relatos vibrantes y a todo color de la sociedad y la historia: una visión dualista y desencarnada del agente, construida por una mente activa montada en un cuerpo ausente, inerte y mudo; una noción aplanada y negativa de estructura interpretada como un conjunto de restricciones externas; y una comprensión idealista del conocimiento como compuesto de trozos de información y conjuntos de representaciones. Estas tres concepciones se refuerzan mutuamente y se unen para literalmente sacar la vida a la vida social, dejándonos con una comprensión incompleta e inadecuada de lo social como un dominio conativo fluido aunque con patrones.

Considero cada uno de estos elementos brevemente. Dadas las concepciones del agente a través de las ciencias sociales polarizadas por una oposición entre homo economicus, la máquina informática racional que maximiza la utilidad individual, descendiente de Bentham y desarrollada por la economía neoclásica, y el homo culturalis, el individuo manipulador de símbolos motivado por normas morales, concepción heredada de Kant y alabada por la antropología cultural, la sociología ha sido torpemente estirada a través de los dos polos. Estos dos modelos imperantes, el "hombre racional" y el "hombre plástico", como los caracterizó Martin Hollis (1977), son igualmente mutilados y mutilantes. ¿Qué es lo que comparten más allá de aparente enfrentamiento? Ambos están descorporalizados y borran del análisis la carne, el deseo y la pasión como una modalidad de existencia social. Estos son los ingredientes de la acción con los que William James discutió y que la psicología profunda de Sigmund Freud intentó capturar, pero solo en un nivel ontogenético. Los hilos encarnados de la ciencia cognitiva contemporánea, que atraviesan la inteligencia artificial, la psicología, la neurobiología, la lingüística y la filosofía, los están redescubriendo rápidamente en el nivel filogenético (Clark 1999, Lakoff y Johnson 1999, Chemero 2013, Shapiro 2014). Pero siguen siendo censurados, ignorados o marginados en los informes científicos sociales estándar.

Sin embargo, "la noción politécnica y polimórfica" puede ser, según Merton (1976), la concepción predominante de la estructura social ubicada directamente fuera del agente, bajo la apariencia de una valla o un embudo, y esto es similarmente cerrado y limitante. Porque las estructuras no existen simplemente como hechos durkheimianos que las personas encuentran en su entorno, en forma de relaciones invisibles, distribuciones objetivas de recursos o sistemas de restricciones y oportunidades que los presionan o los limitan desde fuera. También son redes dinámicas de fuerzas inscriptas sobre y desplegadas en lo profundo del cuerpo como redes perceptivas, habilidades sensoriomotrices, tendencias emocionales $y$, de hecho, como el deseo mismo. Las estructuras son muelles internos o hélices tanto como contenedores externos, vigas o celosías. Son ágiles y están vivas, no inertes ni inmóviles. 
Finalmente, las ciencias sociales trabajan con una noción de conocimiento excesivamente cerebral y pasiva. Otorgamos la dignidad del conocimiento a la información proposicional transmitida por el lenguaje y ubicada en la mente. Pasamos por alto el conocimiento procedimental o práctico adquirido y manifestado en hechos concretos (pragmaticos en griego antiguo significa activo, hábil en asuntos o negocios públicos). Debemos evitar esta concepción que va desde arriba hacia abajo para superar lo que Elizabeth Anscombe (1957) correctamente diagnosticó como la "incorregible concepción contemplativa del conocimiento" heredera de la revolución racionalista, y a partir de allí romper con el concepto idealista (o discursivista) de cultura que le está asociado. Necesitamos reconocer la realidad y la potencia del conocimiento carnal, la comprensión ascendente y visceral del mundo social -en el doble sentido de comprensión intelectual y manejo diestro-que adquirimos al actuar en y sobre él.

¿Qué propiedades del actor humano necesitan ser destacadas para captar este conocimiento práctico corporalizado?

El gran filósofo neo-kantiano Ernst Cassirer (1944) tenía razón al caracterizar al "hombre" como un "animal simbólico" y ver en el lenguaje, el mito, el arte, la religión y la ciencia los principales sistemas simbólicos que los seres humanos han inventado para lidiar con y dar forma a su entorno Pero esta propiedad por sí sola no basta para hacer viable una antropología filosófica. Lo complementaría con cinco propiedades adicionales, todas ellas comenzando convenientemente con S, entonces lo que podríamos llamar esta visión expandida es la concepción del agente "Seis S"

Además de ser un portador de símbolos, el animal humano es sensible, sufriente, hábil (en el original, skilled), sedimentado y situado. Sensible: el agente no solo está dotado de sentidos, externoceptivos, propioceptivos e interoceptivos; también tiene sentido de lo que capta su sensorium. Es capaz de sentir y ser consciente de sentimientos; y el cuerpo es el medio sintetizador de esta conciencia sensible, como lo muestra el neurobiólogo Antonio Damasio en The Feeling of What Happens (1999). Sufriente: el agente está expuesto a las amenazas y golpes del mundo natural y social; tiene necesidades, anhelos y deseos que no se cumplen; está constantemente sujeto al juicio de los demás y enfrenta la ineludible llegada de la muerte. Como tal, vive en la angustia, desamparo y dolor, y sin embargo, resiste. Hábil: el agente social puede "marcar la diferencia" (el significado original de skil atribuido por los antiguos nórdicos es discernir y ajustar) porque, a través de la experiencia y el entrenamiento, el agente adquiere capacidades para actuar y destreza para hacer las cosas de manera competente. Sedimentado: todos estos elementos, nuestros sentidos, el sufrimiento y las habilidades no aparecen con el nacimiento, ni son genéricos ni se constituyen en una relación solipsista con uno mismo. Antes bien, se implantan, cultivan y despliegan a lo largo del tiempo a través de nuestro compromiso en y con el mundo, y gradualmente se depositan en nuestro cuerpo como el producto estratificado de nuestras variadas historias individuales y colectivas. (Merleau-Ponty [1945], confiando en Husserl, llama al "conocimiento habitual del mundo" alojado en el cuerpo propiamente dicho una "ciencia implícita o sedimentada"). Situado: esta sedimentación está determinada por nuestra ubicación única y nuestras trayectorias en el espacio físico y social, precisamente porque ambos están protegidos y encerrados en la frágil envoltura física de nuestro organismo mortal, que no puede estar en dos lugares en un momento dado, sino que integra los rastros de los muchos lugares que hemos ocupado a lo largo del tiempo.

Por último, estos seis elementos están estructurados y fluyen conjuntamente, así como a través del tiempo. Nuestra concepción del agente, la estructura y el conocimiento necesitan ser radicalmente y completamente temporalizados, como lo impulsó Bourdieu (1980/1990, 98-111) hace mucho tiempo en "La acción del Tiempo". Una vez que reconocemos que la cognición es una actividad situada que crece a partir de una danza enredada del cuerpo, la mente, la acción y el mundo, podemos comenzar a recuperar el conocimiento tácito envuelto en prácticas culturales y sociales, y así enriquecer nuestras descripciones y profundizar nuestras explicaciones. Es necesario poner estos tres ingredientes renovados juntos, un ser encarnado involucrando conocimientos prácticos mientras navega configuraciones activas y móviles de afecto, acción y poderes, y contaremos con los cimientos para una sociología de carne y sangre, capaz de producir información multidimensional, descripciones multicolores de la vida social que captan la vida tal como se desarrolla en realidad, en lugar de los torpes informes en blanco y negro que actualmente leemos en revistas académicas.

¿Qué métodos de investigación recomienda para detectar las dimensiones invisibles de acción, estructura y conocimiento?

En principio, los cuatro métodos principales de las ciencias sociales etnográfico, hermenéutico (que abarcan entrevistas y análisis textual), históricos y estadísticos pueden todos abordar cualquier objeto. Pero está claro que están desigualmente equipados para descubrir los componentes de la práctica que no se articulan, simbolizan ni objetivan como tales: las categorías dóxicas, las habilidades fonéticas y las formas ordinarias de ser, sentir y actuar. Un método es el camino real hacia la textura tácita de la acción y la cognición sociales: la observación próxima mediante el involucramiento práctico en la actividad estudiada.

La etnografía -esa técnica particular de producción y 
análisis de datos que se basa en el organismo habilidoso y sensible del observador como principal herramienta de investigación- resulta excepcionalmente adecuada para ayudarnos a incardinarnos en la sociedad, mediante la restauración de las dimensiones praxeológicas de la existencia social. Pero para eso debemos, primero, llegar a una comprensión más clara de la distinción y las virtudes específicas (así como las limitaciones correlativas) de la etnografía como investigación social corporalizada y corporalizante basada en la presencia física con (en) el fenómeno en tiempo y espacio reales y, en segundo lugar, necesitamos reformar nuestra práctica de dos maneras complementarias y aparentemente contradictorias. Por un lado, debemos vincular la etnografía con mayor firmeza a la teoría, contra las ilusiones epistemológicas de la "descripción densa" de Geertz, la ingenuidad filosófica del empirismo al estilo de la Escuela de Chicago y las glamorosas seducciones de la narrativa posmoderna (Wacquant 2002, 1469-71, 1481 -82 y 2009, especialmente 118-122). Por otro lado, necesitamos fomentar formas de participación etnográfica a largo plazo, intensivas, incluso iniciáticas, que permitan al investigador dominar en primera persona, intus et in cute, los esquemas prediscursivos que lo constituyen como miembro competente, diligente y adjunto del universo bajo examen.

Para aprovechar al máximo la etnografía, el sociólogo de campo debe minar y tematizar metódicamente el hecho de que, como todo agente social, llega a conocer su objeto a través del cuerpo; y puede aprovechar la comprensión carnal mediante la profundización de su inserción social y simbólica en el universo que estudia. Esto significa que podemos y debemos trabajar para convertirnos en "observadores vulnerables" en nuestra práctica de trabajo de campo -y no sobre el papel, intentando "escribir de forma vulnerable", inyectando grandes dosis de "subjetividad en etnografía", como lo propuso Ruth Behar (1996, 16 y 6). La estipulación metodológica aquí es sumergirse en la corriente de acción a la mayor profundidad posible, en lugar de mirarlo todo desde la playa; pero con el objetivo de bucear y nadar junto con el método y el propósito, y no con un abandono imprudente que nos llevaría ahogarnos en el torbellino sin fondo del subjetivismo.

En su libro Cuerpo y Alma (Wacquant 2000/2004, nueva edición ampliada 2014) y en los ensayos relacionados, ha tratado de desarrollar lo que denomina una "sociología carnal": ¿qué la diferencia de una etnografía sensual inspirada en la fenomenología?

En pocas palabras, la sociología carnal no es una sociología del cuerpo como objeto sociocultural, sino del cuerpo como fuente de inteligencia social y perspicacia sociológica. Partiendo del hecho bruto de que, como se dijo anteriormente, el agente humano es un ser sensible y sufriente de carne y sangre. (La carne se refiere aquí a la superficie visible del cuerpo vivo mientras que la sangre apunta al circuito interno de la vida que pulsa en lo profundo del cuerpo visceral, como en la revisión de Leder [1990] de Merleau-Ponty. Me sumo aquí a la caracterización de "Quiénes somos" propuesta por George Lakoff y Mark Johnson (1999, 3-4 y passim) en su libro Philosophy in the Flesh, para quien "la mente está encarnada, el pensamiento es en su mayoría inconsciente", y la razón es "en gran medida metafórica e imaginativa", así como, "comprometida emocionalmente"). No se sitúa ni por encima ni por al lado de la acción, sino en su punto de producción. La sociología carnal se esfuerza por evitar el punto de vista del espectador y procura comprender la acción-en-suconstrucción, no la acción-ya-realizada. Su objetivo es detectar y documentar el despliegue de los esquemas prácticos que modulan a la práctica: la construcción de los bloques cognitivos, conativos y afectivos del habitus, cuya estratificación y operaciones están totalmente abiertos a la investigación empírica (Wacquant 2014a, 2014b). Se diferencia de la etnografía sensual, en tanto estudio de campo de los sentidos, que tiene un notable linaje que abarca desde Simmel, Mauss y Lucien Febvre, hasta Elias y la Lebensphilosophie de Arnold Gehlen y Helmut Plessner, hasta los aspectos contemporáneos de la antropología médica y fenomenológica, en que toma las "formaciones sensoriales" no como su objeto de estudio (como lo hace Howes 2003), sino como su medio de estudio.

La sociología carnal se aplica a cualquier objeto y puede usar una variedad de métodos siempre que estos traten al agente social como corporizado e integrado. Por ejemplo, los practicantes de Alltagsgeschichte, microhistoria y la reciente historiografía de las sensibilidades con frecuencia entran en su ámbito, aunque es posible que no lo sepan o no lo intenten. Cuando Carlo Ginsburg (1976) reconstruye el cosmos vívido del molinero del siglo XVI Menocchio antes de ser quemado en la hoguera por ser un presunto hereje en /l formaggio e i vermi; cuando Alf Lüdtke (1993) rastrea las raíces sociales y los efectos de Eigensinn, la obstinada "auto-voluntad" alimentando estrategias recalcitrantes a medio camino entre la acomodación y la resistencia al poder entre los trabajadores alemanes en la primera mitad del siglo XX; cuando Alain Corbin (1988) mapea las cambiantes culturas sensoriales y epistémicas que convirtieron al mar de un objeto de miedo revulsivo en un atractivo lugar de contemplación y engendraron una nueva postura hacia la "naturaleza", están haciendo sociología histórica carnal.

Para los objetos contemporáneos, el mejor método es lo que ahora llamo etnografía enactiva, es decir, la inmersión en el trabajo de campo a través de la que el investigador actúa (elementos de) el fenómeno para despegar las capas de sus propiedades invisibles y para probar sus mecanismos operativos. Adapto el término enactiva de mi colega de Berkeley, el filósofo Alva Noë en su libro Action in Perception (2004, 2), en el que propone que la percepción es "una actividad hábil por parte del animal en su conjunto", que resulta ser una caracterización muy 
adecuada del etnógrafo en el trabajo de campo (Noë mismo toma prestado el adjetivo de la influyente teoría de la "consciencia corporizada" de Francisco Varela, Evan Thompson y Eleanor Rosch [1991]). El primer mandamiento de la indagación corporizada, entonces, es ingresar al teatro de acción con una capacidad ordinaria $y$, al grado más profundo posible, convertirse en aprendiz de las formas en las que viven las personas estudiadas, ya sean pugilistas, profesores o procuradores fiscales, para ganar una aprehensión visceral de su universo material y un trampolín para su reconstrucción analítica.

Pero, ¿qué pasa con aquellas situaciones en las que, por razones prácticas, legales o morales, no puedes convertirte en el fenómeno?

Por supuesto, no siempre es fácil ni sencillo tener acceso y actuar en la escena social analizada: convertirse en un miembro activo a menudo lleva tiempo, requiere tener cualidades especiales u obtener certificación. Puede que no poseas la sangre fría necesaria para ser un oficial de policía o la agilidad para ser una bailarina, no puedes esperar convertirte en juez por poco tiempo, y con suerte no estarás autorizado a realizar una cirugía cerebral en un hospital de alto nivel para propósitos de la comprensión sociológica. Pero bien podrías convertirse en un técnico de utilería para el ballet, inscribirse como pasante con servicios previos al juicio o trabajar como asistente en el pabellón de operaciones. Siempre hay puertas múltiples en cualquier escenario "donde está la acción" -para invocar el ensayo clásico de Goffman (1967) por ese título- y esto proporciona variadas oportunidades para experimentar, y así experimentarse con los componentes del fenómeno aprendiendo la parte y tomando la posición de uno u otro protagonista en el drama social en cuestión, aún si se trata de un drama menor.

Con demasiada frecuencia, los sociólogos no ingresan en la puerta porque ni siquiera la tocan para comprobar qué tan adentro pueden ser invitados. Un ejemplo: hay docenas de monografías de campo sobre personas sin hogar, algunas de ellas bastante admirables; en estilos muy diferentes, recomiendo Snow y Anderson's (1993) Down on their Luck, Bourgois y Schoenberg's (2009) Righteous Dopefiend, y Gowan (2010) Hobos, Hustlers y Backsliders. Sin embargo, ninguno de sus autores ha pasado largos periodos durmiendo en las calles, acurrucado en un hotel SRO para migrantes o pobres (por las siglas en ingles para Single Room Occupancy hotels) o ingresando en un refugio de la ciudad, por no mencionar circulando entre estos tres, que junto con la cárcel local y el hospital público de la ciudad, forman la red de instituciones interconectadas que manejan efectivamente a las personas sin hogar cuando su estado definitorio se vuelve extremadamente problemático. (Hay que remontarse al estudio en equipo inspirado por la WPA de Sutherland y Locke, Twenty Thousand Homeless Men [1936], para encontrar una monografía en la que dormir en los refugios es un elemento central del diseño de la investigación). El estudiante típico de las personas sin hogar sale de la escena al atardecer, justo cuando se plantea y resuelve el problema práctico central: ¿dónde voy a dormir esta noche? Sin embargo, hay mucho que aprender de las pruebas en persona, noche tras noche, de las diferentes técnicas desarrolladas para hacer, sobre y en las calles, un simulacro de "cama" adecuado para uno mismo, para gestionar las relaciones frágiles, para garantizar un mínimo la seguridad, para dominar los dolores y las emociones, para absorber los olores, para alterar el sentido del tiempo, y así sucesivamente. Tantas facetas del fenómeno pueden revelarse mejor a través del cuerpo, en la misma representación de la falta de vivienda, ya sea deliberada y temporal, en el caso del investigador, porque están entretejidas en la textura misma de la acción urgente pero banal y se vuelven de este modo parcialmente imperceptible e indescriptible para quienes están más expuestos a ella.

La investigación encarnada requiere un poco de energía y mucha persistencia. Además de pasar por alto la naturaleza corporalizada de la acción, el conocimiento y la estructura, una de las principales razones por las que la mayoría de las etnografías recaen en la visión textual o hermenéutica del mundo social alimentada por una postura contemplativa (por definición excluida a quienes practican la "participación con observación") es que sus autores simplemente no persisten en sus esfuerzos para obtener acceso y profundizar su integración. Rápidamente retroceden o se detienen en lugar de avanzar e intentar cada ruta para penetrar en la arena de la acción. Pero la tenacidad vale la pena: a Joan Cassell (1991) se le negó inicialmente la entrada a una sala de cirugía y en lugar de eso aconsejó estudiar a la auxiliar de mujeres, pero a fuerza de obstinación acabó observando cirujanos generales en una docena de hospitales, dos organizaciones de mantenimiento de la salud y una clínica pública que realiza unas 200 operaciones. Cassell había planeado un estudio de 18 meses; terminó pasando 33 meses en el campo, al final de los cuales se le permitió "fregar" y manipular separadores junto con los pasantes. Del mismo modo, Gretchen Purser $(2012,2015)$ pasó casi 3 años siendo contratada por agencias de trabajo diario en el centro de la ciudad de Oakland y Baltimore para generar una descripción corporalizada de las estrategias sociales y las experiencias de trabajadores marginales precarizados en la intersección del mercado de trabajo desregulado, el hiperghetto degradado y el sistema carcelario demasiado grande. Ahora, ¿qué tan probable es que una pequeña mujer vegana, con gafas, conviva entre ex convictos negros en la pelea diaria por "boletos" de un "taller" haciendo los trabajos más degradadas? Sin embargo, ella aguantó y emerge en el otro extremo de este experimento social con una descripción incandescente de la realidad de carne y sangre de la "flexploitation" en el bajovientre hinchado de la economía estadounidense.

La sociología carnal se basa en un silogismo y un reto. El silogismo es el siguiente: si es verdad que el cuerpo no es 
solo un producto socialmente construido, sino también un vector socialmente constructivo de conocimiento, práctica y poder, entonces esto se aplica al cuerpo del sociólogo como investigador. El desafío es superar dos milenios de "desprecio por el cuerpo", para citar a Nietzsche, que nos han llevado a interpretar el organismo sensible como un obstáculo para el conocimiento y convertirlo en un recurso central para la investigación científica social. Desplegar metódicamente el propio cuerpo como un instrumento inteligente de producción de conocimiento práctico acelera la adquisición de la competencia social básica -la capacidad operante de sentir, pensar y actuar como lo que sea entre los lo que sea-que, a su vez, ofrece un mejor criterio que la saturación de datos para decidir cuándo has cumplido tu misión.

\section{¿Es la "etnografía enactiva" una nueva forma de} practicar el oficio?

Me siento tentado a decir, por el contrario, que es una etnografía antigua y venerable, que remonta a sus orígenes y edad de oro, en los que hizo hincapié en "aprender haciendo" a través de un contacto duradero e íntimo con "los nativos", en su Poética, Aristóteles nos recuerda que los humanos adquieren su primer conocimiento a través de la mimesis. La etnografía enactiva no hace más que explicitar y luego intensificar las dos características distintivas de cualquier etnografía digna de ese nombre: que el investigador está corporalizado y se ha incorporado a la estructura social y simbólica examinada. Otra forma de caracterizarlo es afirmar que utiliza el habitus como objeto y método de análisis (Wacquant 2011): el investigador descubre y prueba la pertinencia de los esquemas prácticos adquiriéndolos en acto, centrándose intensamente en las técnicas pedagógicas por las cuales son forjados o diseccionados los diseños prácticos. En ese sentido, la etnografía enactiva va en contra de las corrientes de la antropología contemporánea que se han obsesionado tanto con los tropos, la posicionalidad, la ética, la apertura y la multiplicidad de sitios (o "para-sitios"1 alojados en "las complejidades de nuestro tiempo", y no es una broma), y las debilidades de la profesionalización que han alcanzado un estado de parálisis etnográfica debido al meta-análisis (Faubion y Marcus 2009).

Anteriormente propuse que, siempre que la configuración práctica lo permita, podemos y debemos "devenir nativos armados, y retornar" (Wacquant 2009: 119), en una rectificación deliberada a la visión prevaleciente del trabajo de campo en las ciencias sociales dominantes. Ahora deseo enmendar esta formulación porque "volverse nativo" es un eslogan ambiguo que nos lleva a una posición epistémica equivocada. ¿Cuál es el "punto de vista del nativo" que Malinowski (1922/2014) canonizó en Los argonautas del pacífico sur y que Geertz (1974) nos invitó a honrar como el punto de vista de la etnografía?

\footnotetext{
${ }^{1} \mathrm{NT}$ : el autor juega con la expresión "sites", sitios, y "para-sites",
} parásitos
¿De quién es ese punto de vista y en qué momento en el tiempo está tomado? Incluso en una sociedad de pequeña escala basada en el parentesco que se aproxima a la "solidaridad mecánica" de Durkheim, como las Islas Trobriand, existe diferenciación social y jerarquía. De hecho, Malinowski (1922/2014) enfatiza las distinciones de rango y poder entre distritos, tribus y clanes totémicos. Obviamente, el punto de vista del jefe del pueblo no es el de un plebeyo; la perspectiva y las disposiciones de un anciano viudo de un linaje de alto rango no son las mismas que las de una adolescente soltera de un clan totémico inferior. En cualquier sistema de acción hay una división del trabajo de un modo tal que existen puntos de vista, en plural, como puntos de vista captados sobre puntos en evolución en la estructura objetiva del espacio social local. Además, hay una lucha, en cualquier momento, en pos de determinar qué propiedades y posiciones pueden ser calificadas como "nativas". ¿Quién es y quién no es un miembro de buena fe? La respuesta a esta pregunta siempre está en juego en el mundo social en sí mismo $y$, por lo tanto, no debe resolverla el analista por escrito sobre el papel. Como muestro en The Prizefighter's Three Bodies (Los tres cuerpos del boxeador), cualquier punto de vista singular, como el pugilístico, es siempre una construcción sintética selectiva del analista que captura un momento en este concurso dinámico de perspectivas situadas, no una inducción empírica pura desde la observación etnográfica (Wacquant, de próxima publicación).

En su libro más importante Meditaciones Pascalianas, Bourdieu (1997/2000) profundiza su teoría del conocimiento a través del dialogo en las sombras con Pascal. ¿Qué debemos hacer con respecto a esta filiación de Bourdieu con el autor de Les Pensées (1670/1976)?

Esta conexión ha sorprendido a muchos, porque Pascal es un pensador oscuro y enigmático que, además de sus contribuciones a la geometría y la teoría de la probabilidad, es mejor conocido como un apologista del cristianismo, un pensador del abismo existencial y un maestro de la prosa. Pocos científicos sociales lo han encontrado en sus tribulaciones intelectuales y Bourdieu raramente lo menciona abiertamente en sus escritos. Pero la conexión opera y anida en varios niveles. En primer lugar, es una referencia profiláctica destinada a evitar falsas genealogías (acusaciones macartistas de tardías acerca del cripto-marxismo de Bourdieu) y malas lecturas (Bourdieu como defensor de una visión estratégica de la acción en el esquema de la teoría de la elección racional, de la cual era un oponente infatigable). A continuación, es un guiño irónico a la filosofía del sujeto como conciencia soberana, que va de Descartes a Sartre mediada por Husserl (y sus Meditaciones cartesianas, cuyo título Bourdieu parafrasea), con el que el sociólogo francés cruzó espadas durante años. Con Leibniz y especialmente Spinoza, otra gran inspiración de Bourdieu y enérgica defensora de una concepción monista de la actividad humana, Pascal representa el ala no cartesiana de la revolución racionalista, que el autor de La Distinción 
procuró reforzar y extender.

Pero sobre todo hay una profunda afinidad en el nivel de la antropología filosófica y la ontología social: la criatura humana es un ser sufriente, atrapado en y por el mundo, que se arranca del absoluto absurdo de su condición, atrapada "entre el infinito y la nada", a través del conocimiento y la acción, aun cuando este conocimiento nunca pueda ser fundamentado. Este ser y este mundo están amasados por antinomias, parejas jerárquicas que pueden ponerse cabeza abajo: el humano es a la vez "un ángel y una bestia", sometido a los determinismos del universo y capaz de escapar de ellos por el simple hecho de que puede conocerlos y así conocerse a sí mismo; las instituciones se presentan como fundadas en la naturaleza cuando no son más que "personalizadas" ("esta segunda naturaleza destruye la primera"); el orden social parece necesario incluso si es fundamentalmente contingente. La arbitrariedad de las jerarquías que dependen "de los caprichos de los hombres" y la inconmensurabilidad de los poderes ("el orden de los cuerpos, el orden de las mentes, el orden de la caridad"), la centralidad de lo simbólico, el papel del engaño y la creencia (en el sentido de la fides, la fe de que las cosas son como parecen ser: "negar, creer y dudar, bien, estos son para los hombres lo que es galopar para los caballos") como resortes de acción y cemento de la vida colectiva: Pascal le ofrece a Bourdieu no un sistema -el autor de Les Provinciales nunca produjo uno- sino pilares y palancas para "confrontar en su verdad el enigma de la ficción y el fetichismo" (Bourdieu 1997/2000, 6) que están en la base de la sociedad y por lo tanto operar una triple historización, del ser social, del mundo social que lo hace y que él hace, y del conocimiento que puede producir en torno a él.

¿Es posible recurrir a Pascal para profundizar nuestra comprensión de las múltiples formas en que el conocimiento tácito opera en la vida cotidiana tanto aquel que está velado como el que permanece desvelado? ¿Puede ayudarnos a desarrollar una sociología de lo implícito?

"La sensibilidad del hombre a las cosas pequeñas y su insensibilidad a grandes cosas, signo de una extraña inversión", escribió Pascal (1670/1976), y sin embargo, "qué otra cosa es la condición humana." Bourdieu recluta al filósofo jansenista sobre todo para avanzar en su pensamiento sobre el poder simbólico, sus modalidades y efectos, y para repensar el reconocimiento como un conmutador de la existencia social. Esto lo lleva a concebir la tríada conceptual de la cognición, el reconocimiento y la falta de reconocimiento en la vida cotidiana, así como en el funcionamiento institucional en el epicentro de su ontología social. Uno puede ir un paso más allá y desplegar la conflictiva pareja de las "razones del corazón y de la razón" al tomar seriamente las creencias profanas y desarrollar una microsociología política fusionando racionalidad y sentimientos. Del mismo modo, con el concepto de "entretenimiento" (divertissement) y su corolario de que "es la persecución, y no la captura" lo que las personas buscan en cualquier actividad, Pascal abre las puertas de una sociología de la pasión como una modalidad de nuestra relación con el mundo, que implica amor, deseo y sufrimiento, que puede asumir una variedad infinita de formas (filosóficas, políticas, pugilísticas, amorosas, etc.).

Finalmente, al comienzo de sus Pensées, Pascal aborda una distinción sutil pero iluminadora entre el "esprit de géométrie" y el "esprit de finesse", dos modalidades de razonamiento sobre las que los científicos sociales deberían reflexionar (véase Force [2003] para un punteo y Pascal [1657/1985] para profundizar). Nuestra mente geométrica procede con una pequeña cantidad de principios para cortar el mundo, deducir con lógica y concluir con claridad; por el contrario, la destreza intelectual se alimenta de una multitud de principios implícitos que se basan en la experiencia y se sustentan en la intuición local y la analogía sensorial. El primer tipo de razonamiento es abstracto y artificial, nacido del entrenamiento especializado de la mente; el segundo es concreto y natural, brota sintéticamente del flujo de la vida y, ahora sabemos por la ciencia cognitiva, enraizada en movimientos sensomotrices. Pascal compara el espíritu geométrico con la mirada y el espíritu de destreza con el tacto. Esta oposición puede ayudarnos a discernir el dilema particular de las ciencias sociales que exacerba el estudio de lo implícito: la ciencia normal se basa en el espíritu de la geometría, mientras que la vida social se basa en el espíritu de la destreza. La tarea de una ciencia social encarnada, entonces, debe ser conciliar estas dos formas divergentes de razón. "Somos autómatas tanto como mentes", señala Pascal, y por esa razón debemos esforzarnos por evitar "dos excesos: excluir la razón, o admitir solo la razón". No es un mal axioma para iniciar una sociología de la carne y la sangre.

Agradezco a Frank Adolff, Javier Auyero, Sarah Brothers, Megan Comfort, Magnus Hörnqvist, Nazli Ökten y Sohn Searle por sus rápidas reacciones y agudas sugerencias sobre este ensayo y sus argumentos.

\section{Referencias}

Adloff, Frank, Gerund Katharina, y Kaldewey (eds.). 2014. En Revealing tacit knowledge: Embodiment and explication. Berlin: Transcript Verlag

Anscombe, G.E.M. 1957/2000. Intention. Cambridge: Harvard University Press.

Auyero, Javier (ed.). 2005. Número especial Qualitative Sociology sobre Cuerpo y Alma. Qualitative Sociology 28(2): 141-211.

Behar, R. 1996. The vulnerable observer: Anthropology that breaks your heart. Boston: Beacon.

Bourdieu, Pierre. 1980/1990. Le Sens pratique. Paris: Éditions de Minuit (El Sentido práctico, Siglo XXI Editores: México).

Bourdieu, Pierre. 1997/2000. Méditations pascaliennes. 
Paris: Éditions du Seuil (Meditaciones pascalianas, Anagrama: Barcelona).

Bourgois, Philippe, y Jeff Schonberg. 2009. Righteous dopefiend. Berkeley: University of California Press.

Cassell, J. 1991. Expected miracles: Surgeons at work. Filadelfia: Temple University Press.

Cassirer, E. 1944. An essay on man. New Haven: Yale University Press.

Chemero, A. 2013. Radical embodied cognitive science. Review of General Psychology 17(2): 145-150.

Clark, A. 1999. An embodied cognitive science? Trends in Cognitive Sciences 3(9): 345-351.

Corbin, Alain. 1988. Le Territoire du vide. L'Occident et le désir du rivage, 1750-1840. Paris: Aubier.

Damasio, A.R. 1999. The feeling of what happens: Body and emotion in the making of consciousness. New York: Harcourt Brace.

Faubion, J.D., y G.E. Marcus (eds.). 2009. Fieldwork is not what it used to be: Learning anthropology's method in a time of transition. Ithaca: Cornell University Press.

Force, P. 2003. Géométrie, finesse, et premiers principes chez Pascal. Romance Quarterly 50(2): 121-130.

Geertz, Clifford. 1974. "From the native's point of view": On the nature of anthropological understanding.

Bulletin of the American Academy of Arts \& Sciences 26-45 (reeditado en: Local knowledge: Further essays in the interpretation of cultures, 55-7, Nueva York, Basic Books).

Ginzburg, C. 1976. Il formaggio e i vermi. Turín: Einaudi.

Goffman, E. 1967. Interaction ritual: Essays on face-to-face behavior. Nueva York: Anchor.

Gowan, T. 2010. Hobos, hustlers, and backsliders: Homeless in San Francisco. Minneapolis: University of Minnesota Press.

Howes, D. 2003. Sensual relations: Engaging the senses in culture and social theory. Ann Arbor: University of Michigan Press.

Hollis, M. 1977. Models of man: Philosophical thoughts on social action. New York: Cambridge University Press.

Lakoff, G., y M. Johnson. 1999. Philosophy in the flesh: The embodied mind and its challenge to Western Thought. Nueva York: Basic books.

Leder, D. 1990. Flesh and blood: A proposed supplement to Merleau-Ponty. Human Studies 13(3): 209-219.

Lüdtke, A. 1993. Eigen-Sinn: Fabrikalltag, Arbeitererfahrungen und Politik vom Kaiserreich bis in den Faschismus. Hamburgo: Ergebnisse.

Malinowski, B. 1922/2014. Argonauts of the western Pacific: An account of native enterprise and adventure in the archipelagoes of Melanesian New Guinea. Londres: Routledge.

Merleau-Ponty, M. 1945/1962. Phénoménologie de la perception. Paris: Gallimard.

Merton, R. 1976. Structural analysis in sociology. En Approaches to the study of social structure, ed. P. Blau, 21-52. Londres: Open Books.

Noë, A. 2004. Action in perception. Cambridge: MIT Press.

Pascal, B. 1657/1985. De l'Esprit géométrique. Paris: GarnierFlammarion.

Pascal, B. 1670/1976. Pensées. Paris: Garnier-Flammarion.

Purser, G. 2012. "Still doin' time": Clamoring for work in the day labor industry. Working USA: The Journal of Labor \& Society 15(3): 397-415.

Purser, G. 2015. Labor on demand: Dispatching the urban poor. Berkeley: University of California Press.

Searle, J. 2009. Making the social world: The structure of human civilization. Nueva York: Oxford University Press.

Shapiro, L. (ed.). 2014. The Routledge handbook of embodied cognition. Londres: Routledge.

Snow, D.A., y L. Anderson. 1993. Down on their luck: A study of homeless street people. Berkeley: University of California Press.

Stam, H J. (ed.). 2009. Special section on habitus in the work of Loïc Wacquant. Theory \& Psychology 19(6): 707-777.

Sutherland, E y H. J. Locke. 1936. Twenty thousand homeless men: A study of unemployed men in the Chicago shelter. Chicago: Lippincott (re-edición Nueva York, Arno Press, 1971).

Varela, F., E. Thompson y E. Rosch. 1991. The embodied mind: Cognitive science and human experience. MIT press.

Wacquant, L. 2000/2004. Body and soul: Notebooks of an apprentice boxer. Nueva York: Oxford University Press. Nueva edición ampliada 2014.

Wacquant, L. 2002. Scrutinizing the street: Poverty, morality, and the pitfalls of urban ethnography. American Journal of Sociology 107(6): 1468-1532.

Wacquant, L. 2009. The body, the ghetto and the penal state. Qualitative Sociology 32(1): 101-129.

Wacquant, L. 2011. Habitus as topic and tool: Reflections on becoming a Prizefighter. Qualitative Research in Psychology 8(1): 81-92

Wacquant, L. 2014a. Homines in extremis: What fighting scholars teach us about habitus. Body \& Society 20(2): 3-17.

Wacquant, L. 2014b. Putting habitus in its place: A response to the symposium. Body \& Society 20(2): 118- 139.

Wacquant, L. The Prizefighter's three bodies: Steps to a carnal sociology. Nueva York: Oxford University Press. En prensa. 